\title{
Rupture of Aortic Sinus Aneurysms Diagnosed by Left Ventricular Opacification A Case Report
}

\author{
Yan-li Zhang, ${ }^{1}$ MS, Xiao-cong Wang, ${ }^{1} \mathrm{MD}$, Xiao-dong Li, ${ }^{1} \mathrm{MD}$, Cong $\mathrm{Hu},{ }^{2} \mathrm{MS}$, Li-ping Pei, ${ }^{1} \mathrm{MS}$, \\ Wei Yu, ${ }^{1}$ MS, Yan Ma, ${ }^{1}$ MS and Shu Jiang, ${ }^{1}$ MS
}

\begin{abstract}
Summary
Rupture of aortic sinus aneurysms is a rare cardiac malformation that is commonly observed in the right coronary sinus but is rarely observed in the noncoronary sinus. Here, we report a case of aneurysm of the aortic sinus that ruptured into the left ventricular outflow tract and was diagnosed with left ventricular opacification. Left heart echocardiography can clearly demonstrate the structure of the heart and is one of the important diagnostic methods for diagnosing ruptured aortic sinus aneurysms. This observes the perfusion sequence of blood flow to clearly reveal the source, direction, and location of the ruptured aortic sinus aneurysm.
\end{abstract}

(Int Heart J 2020; 61: 186-190)

Key words: Echocardiography, Ruptured aneurysm, Myocardial contrast echocardiography

$\mathrm{L}$

eft heart contrast echocardiography can be classified into left ventricular opacification (LVO) and myocardial contrast echocardiography (MCE). With the expansion of its applications, left heart contrast echocardiography has become one of the hotspots in ultrasonography. LVO can visualize blood flow in the left heart chamber without obvious echogenic reflection, thereby clearly demarcating the endocardial border, making it more conducive to observe ventricular cavity structures and wall motion. ${ }^{1)}$ MCE is a technique that utilizes microbubbles as a red blood cell tracer, in order to determine myocardial perfusion. As it can noninvasively evaluate the local myocardial microcirculation, it has become a global hotspot in echocardiography research. A large number of studies have demonstrated that MCE is important in the quantitative evaluation of myocardial perfusion and the estimation of myocardial infarct size and myocardial viability. ${ }^{2-4)}$ Rupture of aortic sinus aneurysms is a rare cardiac malformation that is commonly observed in the right coronary sinus but is rarely observed in the noncoronary sinus (NCS). Here, we report a case of aneurysm of the aortic sinus that ruptured into the left ventricular outflow tract and was diagnosed using left heart contrast echocardiography. Left heart echocardiography can clearly demonstrate the structure of the heart and is one of the important diagnostic methods for diagnosing ruptured aortic sinus aneurysms. This observes the perfusion sequence of blood flow to clearly reveal the source, direction, and location of the ruptured aortic sinus aneurysm.

\section{Case Report}

A 47-year-old male visited the hospital with chief complaints of shortness of breath following exercise for the past two months. The patient has a past history of third-degree atrioventricular (AV) block. On examination, his heart rate and blood pressure were 64/minute and 159/ $77 \mathrm{mmHg}$, respectively. On percussion, heart sounds were detected toward the left side of the chest, and the leftmost point of the heart sound was located $0.5 \mathrm{~cm}$ inside the midline of the left intercostal bone in the fifth intercostal space. On auscultation, a 3/6-stage systolic jet-like murmur and a diastolic water-like murmur could be heard in the aortic valve area. Cardiac ultrasonography using a GE Vivid E9 system revealed an NCS, which measured $61 \times$ $33 \mathrm{~mm}$ and extended to the anterior mitral valve (Figure 1 A and C). An echo near the left ventricular outflow tract was interrupted, and a $4.5 \mathrm{~mm}$ wide break was observed at the top. During the diastolic phase, the blood flow into the left ventricular outflow tract was visible. Furthermore, the mouth of the aortic valve was squeezed, flow velocity increased, and valve regurgitations were detected (Figure $1 \mathrm{~B}$ and D). Doppler ultrasound revealed a maximum flow rate of $229 \mathrm{~cm} / \mathrm{s}$ at the aortic valve, with a pressure gradient of $20 \mathrm{mmHg}$. Color Doppler flow imaging (CDFI) demonstrated severe aortic regurgitation (AR). The mitral valve could open well but could not completely close. CDFI also demonstrated severe mitral regurgitation (MR). The conclusions for the ultrasound were as follows: (1) possible interlining hematoma between the NCS and mi-

From the ${ }^{1}$ Department of Echocardiography, The First Hospital of Jilin University, Changchun, China and ${ }^{2}$ Prenatal Diagnostic Center, The First Hospital of Jilin University, Changchun, China.

Address for correspondence: Xiao-cong Wang, MD, Department of Echocardiography, The First Hospital of Jilin University, 71 Xinmin Street, Changchun 130000, China. E-mail: zhangyanli381147@163.com

Received for publication May 28, 2019. Revised and accepted September 27, 2019.

Released in advance online on J-STAGE December 26, 2019.

doi: 10.1536/ihj.19-271

All rights reserved by the International Heart Journal Association. 

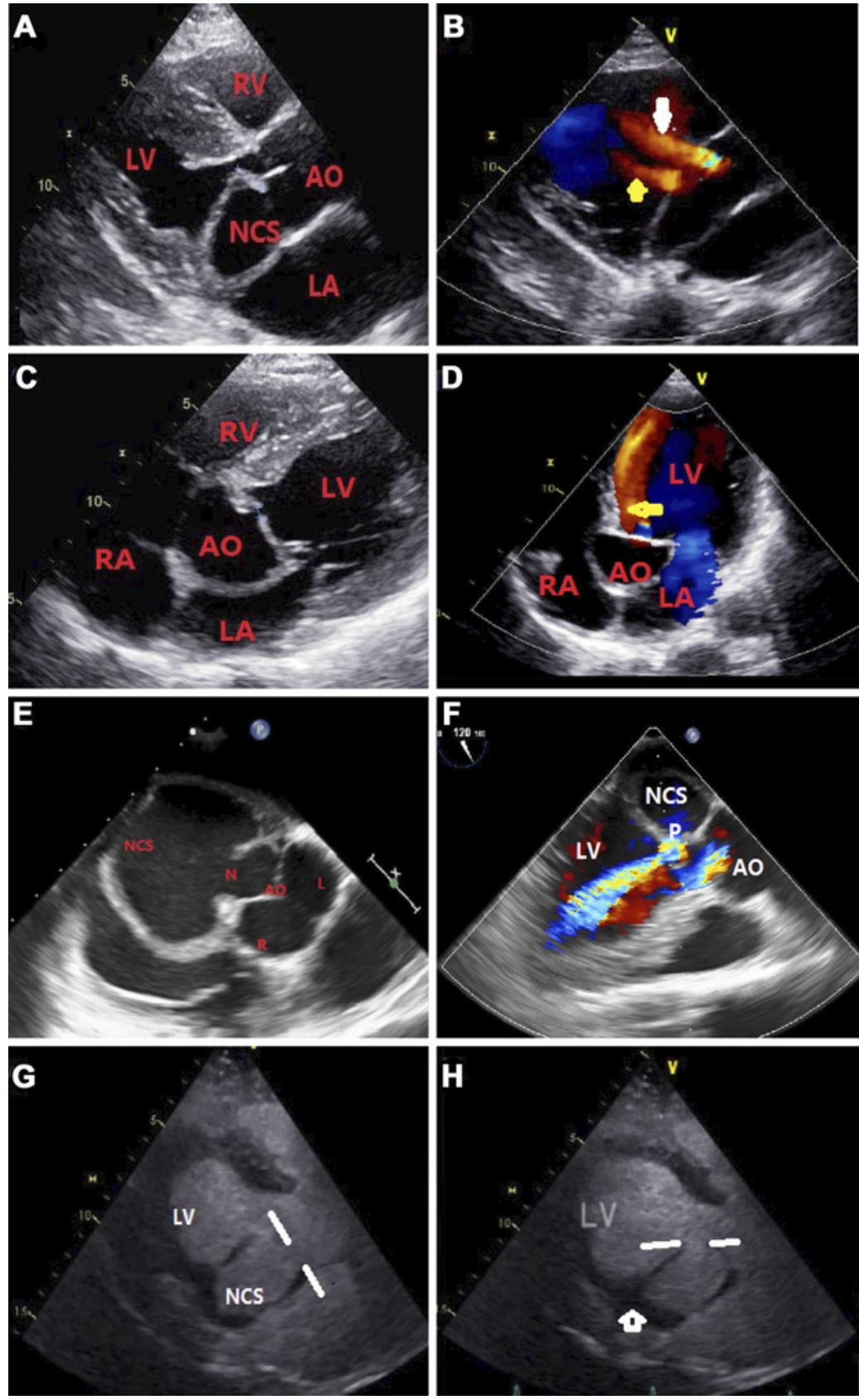

Figure 1. A: Long-axis view of the left ventricle revealing that the aorta has no posterior sinus bulge, forming a cystic structure, that is, NCS. B: Left ventricular long-axis section (CDFI) revealing AR (white arrow) and an unsupervised sinus tumor that broke into the left ventricular outflow tract (yellow arrow). C: Nonstandard five-chamber view. The NCS was bulging into the anterior mitral side. D: Five-chamber view of the heart (CDFI): AR (yellow arrow). E: Transesophageal echocardiography. The short axis of the aorta revealed that the NCS was bulging outward, forming a tumor-like structure (NCS). F: Transesophageal echocardiography. The left ventricular long-axis view reveals the NCS tumor rupture in the left ventricular outflow tract (P). G: LVO. The left ventricular long-axis view reveals that the angiography agent entered the NCS through the ascending aorta (the ascending aorta and NCS traffic port between the two white lines). H: LVO. The left ventricular long-axis section reveals the contrast agent entering the left ventricular outflow tract via the NCS (the NCS is in the left ventricular outflow tract between the two white lines), and the NCS cyst wall thrombus formation (white arrow) is visible. RV indicates right ventricle; LV, left ventricle; LA, left atrium; AO, aortic ostium; NCS, noncoronary sinus; P, noncoronary sinus tumor breach in the left ventricular outflow tract; L, left coronary sinus; R, right coronary sinus; and $\mathrm{N}$, noncoronary sinus. 


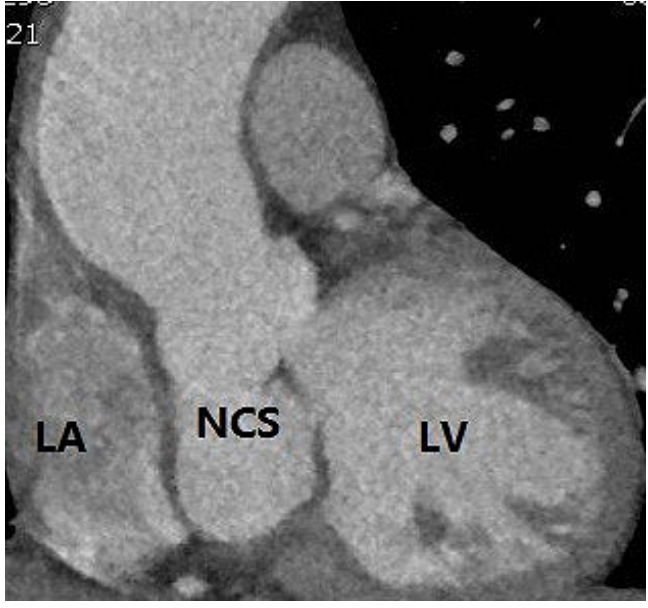

Figure 2. Coronal section of the heart CTA revealing an irregular sac-like downward projection.

tral valve (further examinations are recommended); (2) severe AR; and (3) enlargement of the left atrium, left ventricle, and ascending aorta. On the electrocardiogram, a third-degree $\mathrm{AV}$, borderline escape, and complete right bundle branch block were observed. Coronary computed tomography angiography (CTA) suggested the formation of an intramural coronary artery and a myocardial bridge in the middle part of the anterior descending branch of the left coronary artery. The aortic valve was partially discontinuous, revealing an irregular capsule-like downward projection that is approximately $3.6 \times 5.7 \times 4.1 \mathrm{~cm}$ in size. The ascending aortic lumen was dilated and was approximately $5.4 \mathrm{~cm}$ wide (Figure 2). During the contrastenhanced echocardiography with LVO, $1 \mathrm{~mL}$ of SonoVue was injected into the median cubital vein, followed by 5 $\mathrm{mL}$ of saline. During the test, sequential enhancement of the right atrium-right ventricle-pulmonary artery-left atrium-left ventricle was observed. The contrast agent in the ascending aorta entered the sac (approximately $53 \times$ $35 \mathrm{~mm}$ ) through a gap of approximately $19 \mathrm{~mm}$. This sac was located behind the anterior lobe of the left mitral valve, which protruded into the left ventricular outflow tract. The filling defect in the sac and the rupture (measured as $9 \mathrm{~mm}$ in size) between the sac and the left ventricular outflow tract were visible. In the diastolic phase, some of the contrast agent was observed to run into the left ventricular outflow tract through the rupture (Figure 1 $\mathrm{G}$ and $\mathrm{H}$ ). The MCE revealed no delay in filling of contrast or reduction in the left ventricle segment. Thus, a diagnosis of rupture of an aortic sinus aneurysm (rupturing into the left ventricular outflow tract) was considered. The patient was admitted to the Department of Cardiac Surgery. As the patient also presented with some major vascular changes, Behçet's disease was suspected. However, the patient had no genital ulcers or ocular lesions, and the laboratory tests did not support the diagnosis of Behçet's disease. Hence, Bentall's surgery was planned. Informed consent was obtained from the patient prior to the procedure. The present study was approved by the Ethics Committee of the First Hospital of Jilin University. Preoperative and intraoperative esophageal ultrasound demon-

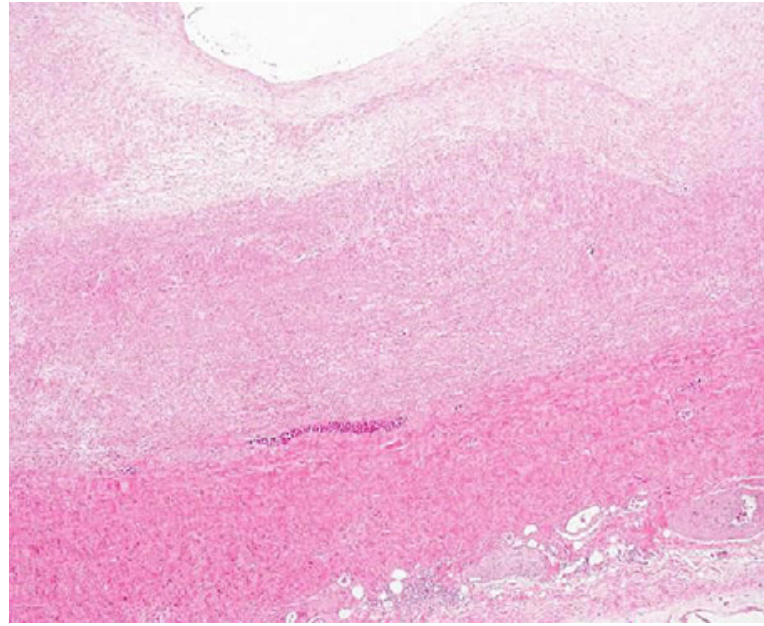

Figure 3. Pathological results of the removed sac during the operation revealing multifocal mucous degeneration of the wall of the ascending aorta, thickening of the intima, tissue hyperplasia of the middle layer, and lymphatic infiltration of the outer layer.

strated the NCS extent to the anterior lobe of the mitral valve, forming an anechoic sac that measured approximately $61 \times 33 \mathrm{~mm}$. Furthermore, there was a discontinued rupture in the anechoic sac, which measured $4.5 \mathrm{~mm}$, beside the left ventricular outflow tract. During the diastolic phase, blood signals were observed to enter the left ventricular outflow tract through the rupture. The flow velocity at the aortic valve increased, and both the aortic and the mitral valves had severe reflux (Figure $1 \mathrm{E}$ and $\mathrm{F}$ ). After the Bentall procedure and mitral mechanical valve replacement surgery, the blood flow in the ascending aorta became smooth, and both the aortic and the mitral mechanical valves performed well. During the cardiovascular surgery, the ascending aortic wall was thick and stiff, and the wall structure was inflamed. In addition, the NCS was obviously enlarged and broke backward into the anterior leaflet of the mitral valve, forming a sac. Some thrombi were noticed in the sac. Furthermore, the sac protruded and broke into the left ventricular outflow tract. The aorta and mitral valve were severely damaged. Thus, the left atrial apex was incised at the NCS, and the thrombi in the sinus and the aortic and mitral valves were removed. As both the aortic and the mitral annuluses were involved, artificial vascular slices were used to reconstruct the aortic and mitral annulus. The opening of the sinus aneurysm in the left ventricular outflow tract was also closed. Pathological examination of the sac (Figure 3 ) revealed the ascending aortic wall with multifocal mucoid degeneration: thickened intima, proliferated fibrous tissue in the middle layer, and lymphatic infiltration scattered in the outer layer.

\section{Discussion}

Aortic sinus tumors are mostly caused by congenital factors that interrupt or soften the muscles and elastic fibers of the aortic sinus wall. The middle layer of the aortic sinus is separated from the annulus. Under hemody- 
namics, the sinus wall gradually becomes thinner and bulges outward to form a cystic substance. ${ }^{5,6)}$ In addition, acquired factors such as infective endocarditis, syphilis, arteriosclerosis, and Marfan syndrome can also lead to aortic sinus aneurysms. ${ }^{7)}$ For the present patient, the investigators considered that the ascending aortic wall with multifocal mucoid degeneration and hypertension both contributed to the formation of the aortic sinus aneurysm and rupture. Aortic sinus aneurysms are most commonly observed in the right coronary sinus, less frequently observed in the NCS, and rarely observed in the left coronary sinus. The broken part is more common in the right ventricle, followed by the right atrium, but rarely in the left atrium and left ventricle, which is classified as type $\mathrm{V}^{8,9)}$ The pressure inside the capsule gradually increases, the wall of the sac becomes thinner and thinned, and finally the sinus aneurysm ruptures into one of the cardiac chambers. When the aneurysm is large, it could compress the surrounding conducting system, such as the AV node or the His bundle, resulting in arrhythmia and conduction block. The aneurysm protrudes into the right ventricular outflow tract, tricuspid valve, and coronary ostium, which causes local obstruction. After the rupture of the sinus aneurysm, the cardiac chamber load is sharply increased, which may lead to the dilatation of the heart chamber and cardiac insufficiency. Furthermore, this could enter into the pericardium, causing tamponade and even death. Owing to the serious clinical consequences of a ruptured aortic sinus aneurysm, surgery should be immediately performed after the diagnosis.

In the present case, the NCS aneurysm ruptured into the left ventricular outflow tract and caused an aortic and mitral valve injury, which is an extremely rare consequence of a ruptured aneurysm. The patient initially underwent conventional echocardiography, which revealed a protruding NCS forming a sac-like structure. This sac involved both the left ventricular outflow tract and the left atrium. However, because of the AR and MR, conventional ultrasound could not clearly demonstrate the NCS aneurysm and position of the rupture. Furthermore, it could not rule out the possibility of an interlining hematoma between the NCS and the anterior lobe of the mitral valve. Left heart echocardiography revealed that the contrast entered the capsular bag immediately after entering the ascending aorta through the left ventricle, and it entered the left ventricular outflow tract through the rupture. Filling defects could be observed in the sac. Hence, it could be concluded that the sac is an aortic sinus aneurysm that broke into the left ventricular outflow tract. Thrombosis presented in the sinus aneurysm, which was consistent with the intraoperative observation. However, MCE examination revealed no significant dysfunction in the coronary microcirculation. In the present case, the contrast did not enter the anterior mitral valve. Hence, the diagnosis of an interlining hematoma was excluded. In addition, the contrast agent could be used to find the echo enhancement and thickening of the anterior and posterior wall of the ascending aorta. Coronary CTA confirmed the above diagnosis and ruled out coronary lesions. Left heart echocardiography played a major role in the diagnosis of the present case. LVO examination could demonstrate the source and path of the aneurysm and determine the rupture location of the sinus. It also could detect the thrombus, which was missed by conventional echocardiography. Furthermore, LVO can improve the image quality of the patients and identify the changes in the anterior and posterior walls of the ascending aorta. ${ }^{10-12)}$ MCE can evaluate coronary microcirculation perfusion and accurately determine disturbances in the coronary microcirculation by assessing the myocardial blood flow. ${ }^{13,14)}$ Left heart echocardiography is important for complicated aortic sinus aneurysms and can reveal the origin of the sinus, its relationship with the surrounding structures, the path of the sinus tumor, and the location of the rupture. Furthermore, it can evaluate the microcirculation function of the coronary artery, in order to provide accurate information for the surgery. ${ }^{15)}$

Aortic sinus aneurysms differ in location and rupture into the heart chamber. Ultrasound manifestations are diverse and can, thereby, be easily missed and misdiagnosed. Hence, these types of aneurysms need to be differentiated from various types of cardiac malformations. ${ }^{16)}$ First, for ventricular septal defects, the rupture of a right aneurysm is usually complicated by ventricular septal defects. In the present case, there was an NCS lesion. Thus, this was easy to differentiate from a ventricular septal defect. Second, for valsalva sinus pseudoaneurysms, these are caused by trauma, but there was no relationship between the pseudoaneurysm and the heart chamber. In the present case, there was no history of trauma. Left heart echocardiography identified a port of communication between the cystic structure and the left ventricular outflow tract. Hence, the diagnosis of valsalva sinus pseudoaneurysm was ruled out. Third, no ruptured coronary sinus in the mitral valve occurred to form a dissection hematoma. Left heart echocardiography revealed that the contrast did not enter the mitral valve, and the mitral valve tissue was separated by dissection. Hence, the diagnosis of dissection of mitral rupture into the mitral valve was ruled out. Finally, Marfan syndrome was considered, which could result in an aortic sinus aneurysm along the lining of the aorta. However, the absence of lesions in the bones and eyes of the patient negated the occurrence of rupture of the aortic sinus tumor caused by Marfan syndrome.

Therefore, two-dimensional echocardiography, CDFI, and spectral Doppler technology can be used to noninvasively and accurately diagnose aortic sinus tumors and determine the location, size, and blood flow of aortic sinus aneurysms. Left heart echocardiography may clearly reveal the structure of the heart, thereby further clarifying the relationship between the aneurysm and the surrounding tissues. Furthermore, it offers the possibility of observing sinus aneurysm breaches, thereby offering a unique diagnostic advantage for patients with difficult-todiagnose aneurysms, such as complex aneurysms.

\section{Disclosure}

Conflicts of interest: None.

Consent: Written informed consent was obtained from the patient for the publication of this manuscript and the ac- 
companying images.

\section{References}

1. Weinsaft JW, Kim RJ, Ross M, et al. Contrast-enhanced anatomic imaging as compared to contrast-enhanced tissue characterization for detection of left ventricular thrombus. JACC Cardiovasc Imaging 2009; 2: 969-79.

2. Main ML, Kusnetzky LL, Dillon D, Daniel WC. Reperfusion assessment using myocardial contrast echocardiography in patients with ST-segment elevation acute myocardial infarction. Am J Cardiol 2004; 93: 1401-3.

3. Korosoglou G, Hansen A, Hoffend J, et al. Comparison of realtime myocardial contrast echocardiography for the assessment of myocardial viability with fluorodeoxyglucose-18 positron emission tomography and dobutamine stress echocardiography. Am J Cardiol 2004; 94: 570-6.

4. Janardhanan R, Burden L, Senior R. Usefulness of myocardia contrast echocardiography in predicting collateral blood flow in the presence of a persistently occluded acute myocardial infarction-related coronary artery. Am J Cardiol 2004; 93: 1207 11.

5. Tosun V, Korucuk N, Guntekin U. Rupture of sinus of Valsalva Aneurysm into left atrium in a patient who is a Candidate for hepatic transplantation. J Cardiovasc Echogr 2018; 28: 130-2.

6. Phatarpekar A, Phadke M, Lanjewar C, Kerkar P. Is the sac waiting to rupture?. Sinus Of Valsalva Anurysm. Aorta (Stam- ford) $2016 ; 4: 105-7$.

7. Ott DA. Aneurysm of the sinus of valsalva. Semin Thorac Cardiovasc Surg Pediatr Card Surg Annu 2006; 165-76.

8. Feldman DN, Roman MJ. Aneurysms of the sinuses of valsalva. Cardiology 2006; 106: 73-81.

9. Xin-Jin L, Xuan L, Bo P, et al. Modified Sakakibara classification system for ruptured sinus of valsalva aneurysm. J Thorac Cardiovasc Surg 2013; 146: 874-8.

10. Lam WC, Pennell DJ. Imaging of the heart: historical perspective and recent advances. Postgrad Med J 2016; 92: 99-104.

11. Nucifora G, Faletra FF. Current applications of contrast echocardiography. Minerva Cardioangiol 2011; 59: 519-32.

12. Wada H, Yasu T, Sakakura K, et al. Contrast echocardiography for the diagnosis of left ventricular thrombus in anterior myocardial infarction. Heart Vessels 2014; 29: 308-12.

13. Zoppellaro G, Venneri L, Khattar RS, Li W, Senior R. Simultaneous assessment of myocardial perfusion, wall motion, and deformation during myocardial contrast echocardiography: A feasibility study. Echocardiography 2016; 33: 889-95.

14. Larsson MK, Da Silva C, Gunyeli E, et al. The potential clinical value of contrast-enhanced echocardiography beyond current recommendations. Cardiovasc Ultrasound 2016; 14: 2

15. Porter TR, Xie F. Contrast echocardiography: latest developments and clinical utility. Curr Cardiol Rep 2015; 17: 569.

16. Weinreich M, Yu PJ, Trost B. Sinus of Valsalva aneurysms: review of the literature and an update on management. Clin Cardiol 2015; 38: 185-9. 